\title{
Atheroprotective effects of statins in patients with unstable angina by regulating the blood-borne microRNA network
}

\author{
SUFANG LI ${ }^{1-3}$, CHENGFU CAO ${ }^{1-3}$, HONG CHEN $^{1-3}$, JUNXIAN SONG $^{1-3}$, CHONGYOU LEE $^{1-3}$, \\ JING ZHANG ${ }^{1-3}$, FENG ZHANG ${ }^{1-3}$, QIANG GENG ${ }^{1-3}$, ZHENG LI $^{1-3}$ and JINGJIN LI ${ }^{4}$ \\ ${ }^{1}$ Department of Cardiology, ${ }^{2}$ Beijing Key Laboratory of Early Prediction and Intervention of Acute Myocardial Infarction \\ and ${ }^{3}$ Center for Cardiovascular Translational Research, Peking University People's Hospital, Beijing 100044; \\ ${ }^{4}$ Department of Cardiology, Beijing Tiantan Hospital, Capital Medical University, Beijing 100050, P.R. China
}

Received May 13, 2016; Accepted March 28, 2017

DOI: $10.3892 / \mathrm{mmr} .2017 .6616$

\begin{abstract}
Experimental studies have demonstrated several effects of statins in acute coronary syndrome (ACS) that may extend their clinical benefit beyond the lipid profile modification itself. However, the precise underlying mechanism remains to be elucidated. microRNAs (miRNAs) serve significant roles in the pathophysiology of atherosclerotic plaque progression. The present study investigated the protective role of statins in patients with unstable angina (UA) by regulating the circulating miRNA network. miRNA array results demonstrated that there were 21 differentially expressed miRNAs in non-statin-treated patients with UA $(\mathrm{n}=8)$ compared with non-coronary artery disease controls $(n=8)$, and 33 differentially expressed miRNAs in statin-treated patients with UA $(n=8)$ compared with non-statin patients. TargetScan and miRanda programs were used to predict miRNAs target genes. miRNAs target genes in vascular endothelial cells and monocytes were clustered based on the CGAP SAGE library via the Database for Annotation, Visualization and Integrated Discovery (DAVID) platform, and miRNA target genes in platelets were clustered based on a UP tissue-specific library via the DAVID platform. The PANTHER database via DAVID platform was used to perform signaling pathway analysis. The miRNA-gene/pathway network was visualized by Cytoscape software. Bioinformatic analysis suggested that statin-induced miRNAs functions were primarily enriched in angiogenesis, integrin and platelet derived growth factor signaling pathways in UA patients. In endothelial cells and platelets, statin-induced miRNAs primarily targeted the integrin signaling pathway, and in monocytes primarily targeted cytoskeletal regulation by the Rho GTPase signaling pathway. These results
\end{abstract}

Correspondence to: Dr Hong Chen, Department of Cardiology, Peking University People's Hospital, 11 Xizhimen South Street, Xicheng, Beijing 100044, P.R. China

E-mail: chenhongbj@medmail.com.cn

Key words: statin, microRNAs, unstable angina, signaling pathways, regulatory network revealed that statins may serve systematic protective roles in UA patients by influencing the circulating miRNA regulatory network. Further studies are required to verify the functions of statin-induced miRNAs in endothelial cells, platelets and monocytes.

\section{Introduction}

Coronary artery disease (CAD) has been a leading cause of mortality and disability worldwide for the past decades, and is likely to remain so for a number of years to come (1). Acute coronary syndromes (ACS) is a high-risk clinical type of CAD which occurs as a result of myocardial ischaemia, and includes acute myocardial infarction and unstable angina (UA). Effective prevention and treatment strategies are important for reducing the morbidity and mortality of CAD. Statins, 3-hydroxy-3-methylglutaryl coenzyme A (HMG-CoA) reductase inhibitors, are the foundation of medical therapy in primary and secondary prevention of cardiovascular diseases. Lipid-lowering therapy uses statins to reduce cardiovascular risk in patients with stable CAD (2) and ACS $(3,4)$. Statin therapy is also recommended (Level of Evidence 1A) by the American College of Cardiology/American Heart Association (ACC/AHA) guidelines for all patients with ACS, regardless of baseline low-density lipoprotein (LDL) levels prior to hospital discharge (5). Although statins were first developed to lower total serum cholesterol and improve the lipid profile, a number of studies have suggested that statins may exert atheroprotective effects beyond cholesterol lowering $(6,7)$, such as improving endothelial function, increasing nitric oxide (NO) activity, reducing oxidative stress, alleviating inflammation, and inhibiting platelet adhesion and the coagulation cascade. Our previous research also demonstrated that statins could improve endothelial function independent of LDL cholesterol reduction (8). All of these results indicated that the clinical benefit of statins in ACS was independent of lipid-reducing effects, but the potential mechanism remains unclear.

microRNAs (miRNAs) are small non-coding RNAs that negatively regulate gene expression at the post-transcription level by combining with target mRNA 3' untranslated region (3'UTR) (9). Single miRNA species can regulate multiple mRNA targets, and single mRNAs may contain several 
miRNA recognition sites on their 3'UTR, which forms a complex regulatory network and controls important biological functions $(10,11)$. Alterations in miRNA levels are associated with numerous human pathologies, including cancer $(12,13)$, and metabolic $(14,15)$ and cardiovascular diseases $(16,17)$. miRNAs have also been investigated in the blood, where they have been detected in plasma, platelets, erythrocytes and nucleated blood cells, and serve as novel diagnostic markers (18). It has also been identified that miRNAs are capable of mediating cell-cell communication transferred by microvesicles, and serve an important regulatory role in a number of diseases (19).

It has been reported that statins are able to serve their biological role by regulating miRNA expression in CAD-associated cells, including platelets (20), endothelial cells (21), endothelial progenitor cells $(22,23)$ and monocytes $(24)$. Statins may enhance the stability of atherosclerotic plaques mediated by miRNAs in UA patients; therefore, the present study aimed to investigate the influence of statins on the circulating miRNA profile in UA patients, and analyzed the miRNA-mediated regulatory network in these patients.

\section{Materials and methods}

Patients. The present study was performed in accordance with the Helsinki Declaration and was approved by the Ethics Review Board of Peking University People's Hospital (Beijing, China). The patients were recruited from Peking University People's Hospital and were as follows: 8 non-statin controls without CAD, as assessed by coronary angiography (group 1: Control group); 8 UA patients with non-statin medication (group 2: UA group, also designated non-statin group); and 8 UA patients with statin treatment (group 3: statin group). All subjects gave their written informed consent. Criteria for the diagnosis of UA were according to the ACC/AHA 2011 guidelines (1). Patients presenting elevated troponin $\mathrm{I}(\geq 0.04 \mathrm{ng} / \mathrm{ml})$ and/or creatine kinase ( $\geq 5 \mathrm{ng} / \mathrm{ml}$ ) or with myocarditis, cardiogenic shock, a history of severe hepatic or renal dysfunction, leukemia, ongoing inflammation and malignant disease, were excluded.

Blood collection and RNA extraction. Blood was collected from each patient via venipuncture into PAXgene Blood RNA tubes (BD Diagnostics, Inc., Sparks, MD, USA) prior to coronary angiography. A PAXgene Blood miRNA kit (Qiagen, Inc., Valencia, CA, USA) was used for RNA isolation according to manufacturer's protocol.

miRNA taqman low density array (TLDA). TLDA was used to determine differentially expressed miRNAs in whole blood from subjects ( $\mathrm{n}=8 /$ group). Approximately $15 \mathrm{ng}$ of total RNA was reverse-transcribed with a Taqman miRNA reverse transcription kit (Applied Biosystems; Thermo Fisher Scientific, Inc., Waltham, MA, USA) and Taqman miRNA Multiplex RT assays (Human Pool A; Applied Biosystems; Thermo Fisher Scientific, Inc.). The reverse transcription products were analyzed using Human MicroRNA TLDA card A version 3.0 (Applied Biosystems; Thermo Fisher Scientific, Inc., Waltham, MA, USA), which can detect the expression of 372 miRNAs simultaneously. miRNAs levels were normalized to the levels of U6. DataAssist software version
3.01 (www.lifetechnologies.com/us/en/home/technicalresources/software-downloads/dataassist-software.html) was used to calculate the relative levels of miRNAs, using the quantitation threshold $(\mathrm{Cq})$ method (25). Significance analysis of microarrays was used to analyze differentially expressed miRNAs between two groups. The criteria for the differentially expressed miRNAs were a fold change $\geq 2$ or $\leq 0.5$, q-value $<0.05$ and a false discovery rate $<0.05$ in comparison between two groups.

Bioinformatic analysis. The target genes of miRNAs were predicted in TargetScan (www.targetscan.org/) and miRanda (miracle.igib.res.in/miracle/) databases, and the target genes simultaneously predicted by the two databases were selected for the next step of signaling pathway analysis. The Database for Annotation, Visualization and Integrated Discovery (DAVID; david.abcc.ncifcrf.gov/) 6.7 platform was used to input target genes, and the enriched pathways of these genes were obtained in PANTHER (www.pantherdb.org/). Clustering of target genes according to different cell types was based on CGAP SAGE (for monocytes and endothelium; cgap.nci.nih.gov/SAGE) and a UP tissue-specific library (for platelets) via the DAVID platform. To visualize the putative target genes or functional pathways of miRNAs, the network dataset was entered in Cytoscape version 3.0.0 beta 1 (www. cytoscape.org).

Statistical analysis. Quantitative data are presented as the mean \pm standard deviation. For continuous variables, statistical significance was calculated using Student's t-test for the comparison of two groups. For categorical variables, statistical significance was calculated using the chi-square test for the comparison of two groups. All tests were two-sided. SPSS version 17.0 (SPSS, Inc., Chicago, IL, USA) was used for all statistical analyses. $\mathrm{P}<0.05$ was considered to indicate a statistically significant difference.

\section{Results}

Regulatory effect of statins on blood-borne miRNA expression profiling in UA patients. To determine the role of statins in UA patients mediated by circulating miRNA, the characteristic of circulating miRNA profiling in non-statin patients with UA $(n=8)$ compared with non-CAD controls $(n=8)$ was first studied (Table I). Under the pathological condition of UA without statin therapy, there were 21 differentially expressed miRNAs; 20 miRNAs were downregulated and 1 miRNA was upregulated (Fig. 1A; Table II). However, 33 upregulated miRNAs were identified in UA patients treated with statin $(n=8)$ compared with non-statin patients (Fig. 1B; Table III). Among the 33 upregulated miRNAs, there were 20 nascent miRNAs and 13 initially downregulated miRNAs in non-statin-treated patients with UA (Fig. 1C), which indicated that statins may alter the circulating miRNA expression profiles of UA patients.

Signaling pathways analysis targeted by differentially expressed miRNAs induced by statin in UA patients. In order to understand the biological role of statins in UA patients, the signaling pathways targeted by 21 differentially expressed miRNAs in non-statin patients with UA were first analyzed. 
Table I. Clinical characteristics of patients.

\begin{tabular}{|c|c|c|c|c|c|}
\hline & \multicolumn{2}{|c|}{ Non-statin } & \multirow{2}{*}{$\frac{\text { Statin }}{\substack{\text { Group } 3 \text { UA } \\
(\mathrm{n}=8)}}$} & \multicolumn{2}{|c|}{ P-value } \\
\hline & $\begin{array}{l}\text { Group } 1 \text { controls } \\
\qquad(\mathrm{n}=8)\end{array}$ & $\begin{array}{l}\text { Group } 2 \text { UA } \\
\quad(n=8)\end{array}$ & & $\begin{array}{l}\text { Group } 2 \text { vs. } \\
\text { Group } 1\end{array}$ & $\begin{array}{c}\text { Group } 3 \text { vs. } \\
\text { Group } 2\end{array}$ \\
\hline \multicolumn{6}{|l|}{ General data } \\
\hline Age (years) & $62 \pm 9$ & $66 \pm 9$ & $58 \pm 8$ & 0.42 & 0.08 \\
\hline Sex (male/female) & $5 / 3$ & $4 / 4$ & $3 / 5$ & 0.61 & 0.61 \\
\hline SBP & $133 \pm 11$ & $136 \pm 15$ & $132 \pm 18$ & 0.76 & 0.67 \\
\hline DBP & $82 \pm 4$ & $79 \pm 6$ & $82 \pm 14$ & 0.24 & 0.53 \\
\hline \multicolumn{6}{|l|}{ Medical history, $\%$} \\
\hline Hypertension & 62.5 & 87.5 & 62.5 & 0.25 & 0.25 \\
\hline Diabetes & 0 & 12.5 & 12.5 & 0.30 & $>0.99$ \\
\hline Hyperlipaemia & 12.5 & 25.0 & 50.0 & 0.52 & 0.30 \\
\hline \multicolumn{6}{|c|}{ Laboratory test, mmol/1 } \\
\hline LDL-C & $2.13 \pm 1.06$ & $2.61 \pm 0.97$ & $2.41 \pm 0.97$ & 0.36 & 0.68 \\
\hline HDL-C & $1.26 \pm 0.72$ & $1.04 \pm 0.10$ & $1.08 \pm 0.24$ & 0.40 & 0.67 \\
\hline $\mathrm{TC}$ & $4.07 \pm 0.90$ & $4.22 \pm 1.07$ & $4.07 \pm 0.82$ & 0.77 & 0.77 \\
\hline $\mathrm{TG}$ & $1.35 \pm 0.61$ & $1.18 \pm 0.38$ & $1.27 \pm 0.78$ & 0.51 & 0.76 \\
\hline Glucose & $6.28 \pm 1.57$ & $4.99 \pm 0.83$ & $5.25 \pm 0.77$ & 0.06 & 0.52 \\
\hline Creatinine & $91.25 \pm 29.20$ & $85.75 \pm 33.04$ & $78.13 \pm 40.75$ & 0.73 & 0.69 \\
\hline \multicolumn{6}{|l|}{ Medication, $\%$} \\
\hline Aspirin & 12.5 & 50.0 & 75.0 & 0.11 & 0.30 \\
\hline Clopidogrel & 12.5 & 25.0 & 50.0 & 0.52 & 0.30 \\
\hline Calcium antagonist & 12.5 & 50.0 & 25.0 & 0.11 & 0.30 \\
\hline ACEI & 0 & 25.0 & 12.5 & 0.13 & 0.52 \\
\hline ARB & 0 & 37.5 & 12.5 & 0.06 & 0.25 \\
\hline$\beta$-blocker & 37.5 & 50.0 & 37.5 & 0.61 & 0.61 \\
\hline
\end{tabular}

Data are presented as the mean \pm standard deviation. P-values represent comparisons between non-statin-treated UA patients and controls or between UA patients treated with and without statins. Comparisons between the two groups were performed with Student's t-test for continuous variables and with chi-square test for categorical variables. SBP, systolic blood pressure; DBP, diastolic blood pressure; LDL-C, low density lipoprotein cholesterol; HDL-C, high-density lipoprotein cholesterol; TC, total cholesterol; TG, triglyceride; ACEI, angiotensin-converting enzyme inhibitor; ARB, angiotensin receptor blocker.

Each miRNA target was entered into the DAVID platform and the signaling pathways referring to these targets were obtained from the PANTHER database. Bioinformatics analysis results demonstrated that the target genes were mainly enriched in the following pathways: Angiogenesis (regulated by miR-15b, 17, 20a, 93, 195, 374-5p, 454), platelet derived growth factor (PDGF) signaling pathway (regulated by let-7 g, 7e, miR-17, 20a, 532-3p), integrin signaling pathway (regulated by let-7e, miR-25, 26a, 532-3p) and p53 pathway feedback loops (regulated by let-7 g, miR-25, 26b, 92a; Fig. 2). There were 16 out of 21 miRNAs involved in the regulation of signaling pathways in non-statin-treated patients with UA. The effects of these pathways were enhanced in unstable coronary heart disease for the downregulation of associated miRNAs, which may contribute to plaque destabilization.

Subsequently, the target pathways of 33 upregulated miRNAs in statin-treated UA patients were analyzed by the same bioinformatic method. The results demonstrated that the target genes of 13 initially downregulated miRNAs were primarily involved in angiogenesis (regulated by miR-15b, 17, 20a, 93), the PDGF signaling pathway (regulated by let-7e, miR-17, 20a, 532-3p) and the integrin signaling pathway (regulated by let-7e, miR-25, 26a, 92a; Fig. 3A). Although 20 nascent miRNAs were primarily involved in angiogenesis (regulated by miR-19a, 19b, 331-3p, 342-3p, 484) and the Wnt signaling pathway (regulated by miR-19a, 19b, 222; Fig. 3B), the first three signaling pathways targeted by 33 statin-induced miRNAs were still the angiogenesis, integrin and PDGF signaling pathways. A total of 25 of 33 miRNAs were involved in regulating these biological pathways in UA patients treated with statins (Fig. 3). The effects of these signaling pathways were inhibited by statins in UA patients by upregulation of associated miRNAs, which suggested the atheroprotective effects of statins in UA patients.

Target genes of statin-induced differentially expressedmiRNAs involved in the angiogenesis, integrin and PDGF signaling pathways. Since the angiogenesis, integrin and PDGF signaling pathways are three of the most important pathways targeted by upregulated miRNAs induced by statins in UA patients, 
A

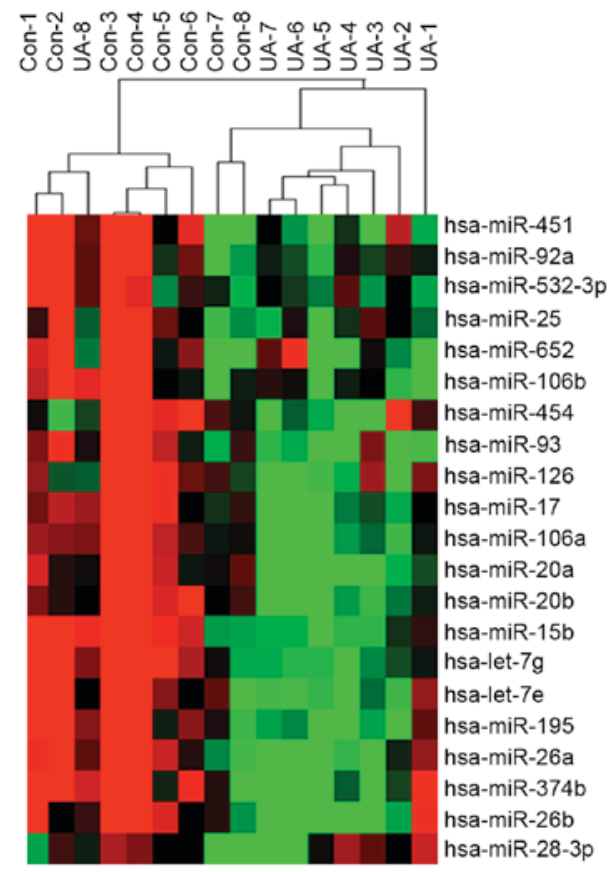

B

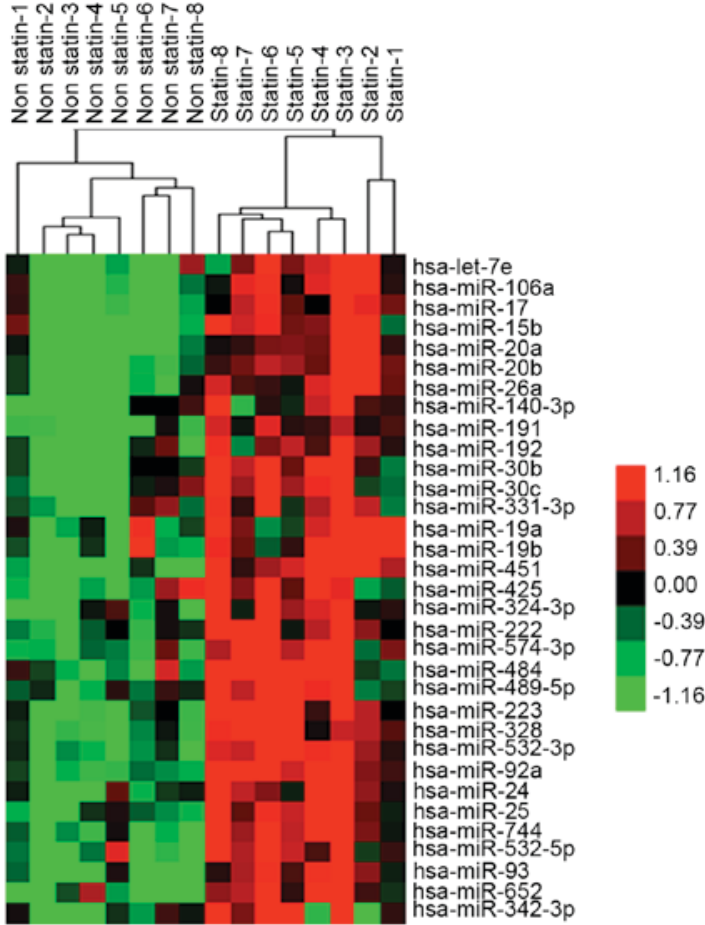

C

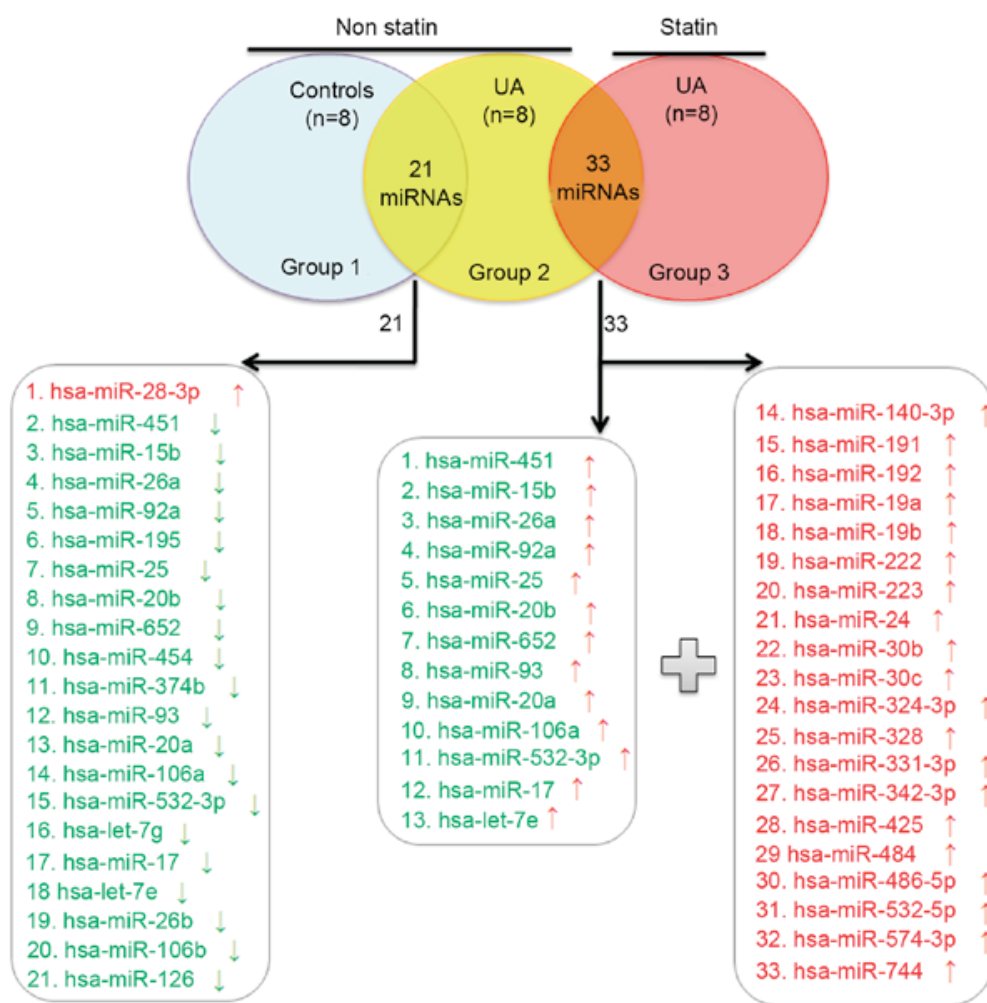

Figure 1. Blood-borne miRNA differential expression profiling in (A) UA patients without statin treatment compared with non-CAD controls and (B) in UA patients with statin treatment compared with non-statin-treated UA patients, as detected by TLDA. (A) RNA was isolated from whole blood of subjects with no statin treatment (UA, $n=8$ vs. Con, $n=8$ ). (B) RNA was isolated from whole blood of UA patients with and with no statin therapy (statin, $n=8$ vs. non-statin, $n=8$ ). The heat map illustrates levels of significantly altered miRNAs. Color intensity is scaled within each row so that the highest expression value corresponds to bright red and the lowest to bright green. (C) There were 33 upregulated miRNAs in the statin group compared with the non-statin group, of which 13 miRNAs were downregulated in the non-statin group compared with the non-CAD group, and 20 nascent miRNAs. miRNA/miR, microRNA; UA, unstable angina; CAD, coronary artery disease; TLDA, taqman low density array; Con, control.

the miRNAs and their target genes involved were analyzed. Each group of target genes of 33 miRNAs was entered into the DAVID platform, and then the angiogenesis, integrin and
PDGF signaling pathways were obtained as well as the relevant genes and miRNAs in the PANTHER database. Bioinformatic analysis revealed that 9 of 33 miRNAs were involved in the 
Table II. Circulating miRNA differential expression profiling in non-statin-treated patients with UA compared with non-CAD controls.

\begin{tabular}{|c|c|c|c|c|}
\hline No. & Gene ID & Score $(d)$ & Fold change (UA/Control) & q-value, $\%$ \\
\hline 1 & hsa-miR-28-3p & 1.71 & 3.77 & $<0.01$ \\
\hline 2 & hsa-miR-451 & -2.54 & 0.32 & $<0.01$ \\
\hline 3 & hsa-miR-15b & -2.47 & 0.21 & $<0.01$ \\
\hline 4 & hsa-miR-26a & -2.21 & 0.28 & $<0.01$ \\
\hline 5 & hsa-miR-92a & -2.07 & 0.41 & $<0.01$ \\
\hline 6 & hsa-miR-195 & -2.02 & 0.09 & $<0.01$ \\
\hline 7 & hsa-miR-25 & -1.92 & 0.47 & $<1.35$ \\
\hline 8 & hsa-miR-20b & -1.89 & 0.08 & $<1.35$ \\
\hline 9 & hsa-miR-652 & -1.88 & 0.39 & $<1.35$ \\
\hline 10 & hsa-miR-454 & -1.86 & 0.18 & $<1.35$ \\
\hline 11 & hsa-miR-374b & -1.80 & 0.23 & $<1.35$ \\
\hline 12 & hsa-miR-93 & -1.78 & 0.21 & $<1.35$ \\
\hline 13 & hsa-miR-20a & -1.76 & 0.13 & $<1.35$ \\
\hline 14 & hsa-miR-106a & -1.75 & 0.20 & $<1.35$ \\
\hline 15 & hsa-miR-532-3p & -1.74 & 0.49 & $<1.35$ \\
\hline 16 & hsa-let-7g & -1.69 & 0.09 & $<1.35$ \\
\hline 17 & hsa-miR-17 & -1.68 & 0.20 & $<1.35$ \\
\hline 18 & hsa-let-7e & -1.67 & 0.16 & $<1.35$ \\
\hline 19 & hsa-miR-26b & -1.64 & 0.16 & $<1.35$ \\
\hline 20 & hsa-miR-106b & -1.63 & 0.32 & $<1.35$ \\
\hline 21 & hsa-miR-126 & -1.62 & 0.17 & $<1.35$ \\
\hline
\end{tabular}

Whole blood miRNA expression profiles demonstrating significant alterations in UA patients $(n=8)$ without statin treatment compared with non-CAD controls $(n=8)$. miRNAs with fold change $\geq 2$ or $\leq 0.5$, q-value $<0.05$ and FDR $<0.05 \%$ are listed. miRNA, microRNA.

angiogenesis pathway referring to 132 target genes (Fig. 4A), 6 of 33 miRNAs in the integrin signaling pathway by targeting 95 genes (Fig. 4B), and 5 of 33 miRNAs in the PDGF signaling pathway including 81 target genes (Fig. 4C). By upregulating miRNA levels, statins may suppress the expression of relevant genes to inhibit the angiogenesis, integrin and PDGF signaling pathways in UA patients.

Signaling pathways analysis in unstable plaque-associated cell types of UA patients treated with statins. Vascular endothelial cells, monocytes and platelets are the main sources of circulating miRNAs in CAD patients (26) and these three cell types are predominantly involved in the formation of unstable plaque and plaque rupture. Therefore, to clarify the role of statin in the formation of unstable plaque, the target pathways of 33 statin-induced miRNAs were analyzed in vascular endothelial cells, monocytes and platelets, separately. Target genes of the 33 miRNAs in the three different cell types were obtained from the CGAP SAGE database (for monocytes and endothelial cells) and a UP tissue-specific database (for platelets). These genes were then entered into the DAVID platform and the signaling pathways in the PANTHER database. The results indicated that differentially expressed miRNAs induced by statins mainly targeted integrin signaling pathways both in vascular endothelial cells (regulated by let-7e, miR-17, 19a, 19b, 20a, 20b, 24, 30b, 30c, 93, 106a, 342-3p, 486-5p) and platelets (regulated by miR-15b, 17, 19a, 19b, 20a, 20b, 24, 25,
93, 30c, 106a, 425, 484; Fig. 5), and cytoskeletal regulation by Rho GTPase pathway in monocytes (regulated by miR-20b, 24 , 93, 106a, 324-3p, 328, 342-3p, 484, 532-3p; Fig. 5). Statins may inhibit atherosclerosis progression by influencing the effects of different signaling pathways in unstable plaque-related cells mediated by miRNAs.

\section{Discussion}

Statins serve an important role in the prevention and treatment of cardiovascular diseases due to their pleiotropic effects. It has been previously reported that statins improve endothelial function in patients with CAD (8), alleviating inflammation in the aorta of hypercholesterolaemic atherosclerotic rabbits (27) and increasing NO synthesis in rat vascular smooth muscle cells (28). Previous studies have demonstrated that statins can influence cellular biological activity by regulating the expression of particular miRNAs. For example, simvastatin can decrease miR-155 expression through interfering with the mevalonate-geranylgeranyl-pyrophosphate-RhoA signaling pathway, and then increasing endothelial nitric oxide synthase expression and endothelium-dependent vasodilation (21). Atorvastatin treatment increased angiogenesis-associated miR-221, miR-222 and miR-92a expression in endothelial progenitor cells (23) and inhibited immune response by downregulating toll-like receptor 4 signaling by inducing let-7i expression in monocytes from CAD patients (24). 
Table III. Circulating miRNAs differential expression profiling in UA patients treated with statin compared with non-statin patients.

\begin{tabular}{|c|c|c|c|c|}
\hline No & Gene ID & Score $(d)$ & Fold change (statin/non statin) & q-value, $\%$ \\
\hline 1 & hsa-miR-191 & 5.52 & 2.28 & $<0.01$ \\
\hline 2 & hsa-miR-92a & 5.15 & 3.28 & $<0.01$ \\
\hline 3 & hsa-miR-223 & 4.92 & 3.17 & $<0.01$ \\
\hline 4 & hsa-miR-532-3p & 4.46 & 2.69 & $<0.01$ \\
\hline 5 & hsa-miR-451 & 4.31 & 4.77 & $<0.01$ \\
\hline 6 & hsa-miR-30b & 4.22 & 2.80 & $<0.01$ \\
\hline 7 & hsa-miR-15b & 4.15 & 3.57 & $<0.01$ \\
\hline 8 & hsa-miR-26a & 3.77 & 3.38 & $<0.01$ \\
\hline 9 & hsa-miR-30c & 3.65 & 2.50 & $<0.01$ \\
\hline 10 & hsa-miR-19b & 3.41 & 2.38 & $<0.01$ \\
\hline 11 & hsa-miR- 25 & 3.39 & 2.92 & $<0.01$ \\
\hline 12 & hsa-miR-222 & 3.34 & 2.54 & $<0.01$ \\
\hline 13 & hsa-miR-574-3p & 3.33 & 2.98 & $<0.01$ \\
\hline 14 & hsa-miR-484 & 3.31 & 2.88 & $<0.01$ \\
\hline 15 & hsa-miR-24 & 3.31 & 2.02 & $<0.01$ \\
\hline 16 & hsa-miR-652 & 3.30 & 3.06 & $<0.01$ \\
\hline 17 & hsa-miR-486-5p & 3.27 & 2.53 & $<0.01$ \\
\hline 18 & hsa-miR-324-3p & 3.20 & 2.22 & $<0.01$ \\
\hline 19 & hsa-miR-331-3p & 3.10 & 2.07 & $<0.01$ \\
\hline 20 & hsa-miR-106a & 3.06 & 3.46 & $<0.01$ \\
\hline 21 & hsa-miR-328 & 3.05 & 2.70 & $<0.01$ \\
\hline 22 & hsa-miR-20a & 3.02 & 4.15 & $<0.01$ \\
\hline 23 & hsa-miR-140-3p & 2.77 & 2.23 & $<0.01$ \\
\hline 24 & hsa-miR-93 & 2.71 & 3.61 & $<0.01$ \\
\hline 25 & hsa-miR-17 & 2.67 & 3.61 & $<0.01$ \\
\hline 26 & hsa-miR-192 & 2.67 & 2.72 & $<0.01$ \\
\hline 27 & hsa-miR-20b & 2.61 & 4.13 & $<0.01$ \\
\hline 28 & hsa-miR-532-5p & 2.55 & 2.74 & $<0.01$ \\
\hline 29 & hsa-miR-744 & 2.54 & 3.68 & $<0.01$ \\
\hline 30 & hsa-miR-342-3p & 2.49 & 2.21 & $<0.01$ \\
\hline 31 & hsa-miR-19a & 2.49 & 2.04 & $<0.01$ \\
\hline 32 & hsa-miR-425 & 2.39 & 2.05 & $<0.01$ \\
\hline 33 & hsa-let-7e & 2.28 & 3.17 & $<0.01$ \\
\hline
\end{tabular}

Whole blood miRNA expression profiles demonstrating significant alterations in statin-treated patients with UA (n=8) compared with non-statin-treated UA patients $(\mathrm{n}=8)$. miRNAs with fold change $\geq 2$ or $\leq 0.5$, q-value $<0.05$ and FDR $<0.05 \%$ are listed. miRNA, microRNA.

However, whether statins serve systematic biological roles in CAD patients by regulating the miRNAs network remains to be elucidated. The present study demonstrated that in UA patients, statins may exert pleiotropic effects in endothelial cells, platelets and monocytes by influencing the blood-borne miRNA regulatory network.

The present study first examined the miRNA expression profile in the whole blood of non-statin-treated UA patients and non-CAD controls. The TLDA results demonstrated that there were 21 differentially expressed miRNAs in non-statin-treated patients compared with controls. The majority of the miRNAs were downregulated and mainly targeted angiogenesis, p53 pathway feedback loops, integrin and PDGF signaling pathways, which suggested the pathological states of UA patients at the molecular level. The function enhancement of the four signaling pathways may partially explain atherosclerotic plaque progression in UA patients (29-35). Nevertheless, compared with the UA patients without statin treatment, there were 33 upregulated miRNAs in statin-treated UA patients. The 33 upregulated miRNAs were composed of 13 initially downregulated miRNAs in non-statin-treated UA patients and 20 nascent miRNAs.

In order to understand the biological role of statin in UA patients, the signaling pathways mediated by the differentially expressed miRNAs were next analyzed. Bioinformatic analysis revealed that the 33 upregulated miRNAs induced by statin were primarily involved in angiogenesis, integrin and PDGF signaling pathways. Consistent with these 


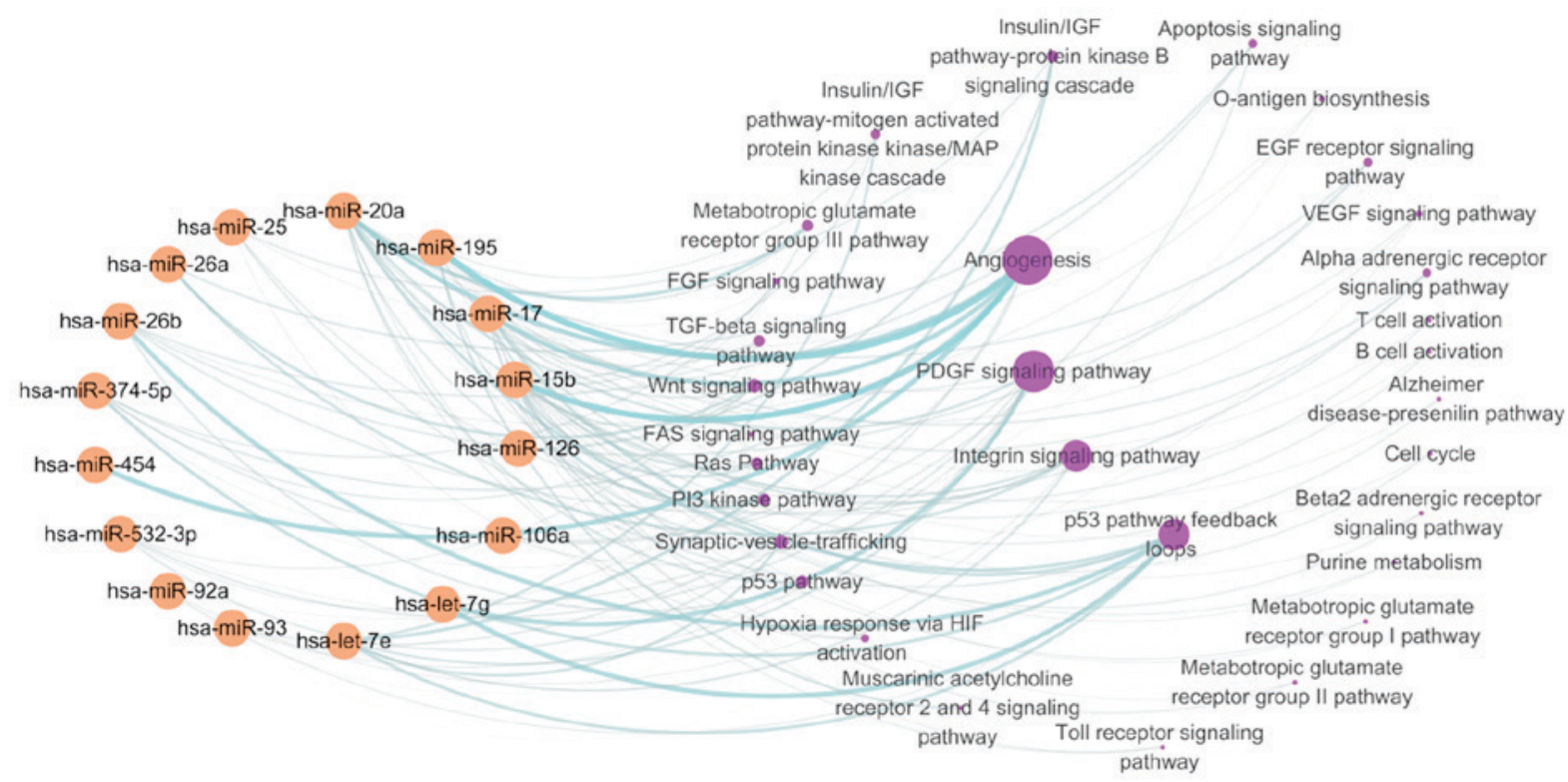

Figure 2. Potential signaling pathways targeted by differentially expressed miRNAs in whole blood of non-statin-treated patients with UA compared with non-CAD patients. The target genes of miRNAs involved in signaling pathways were clustered by DAVID based on PANTHER pathway. The network was generated by the Cytoscape tool. miRNA/miR, microRNA; UA, unstable angina; CAD, coronary artery disease; DAVID, Database for Annotation, Visualization and Integrated Discovery.

A

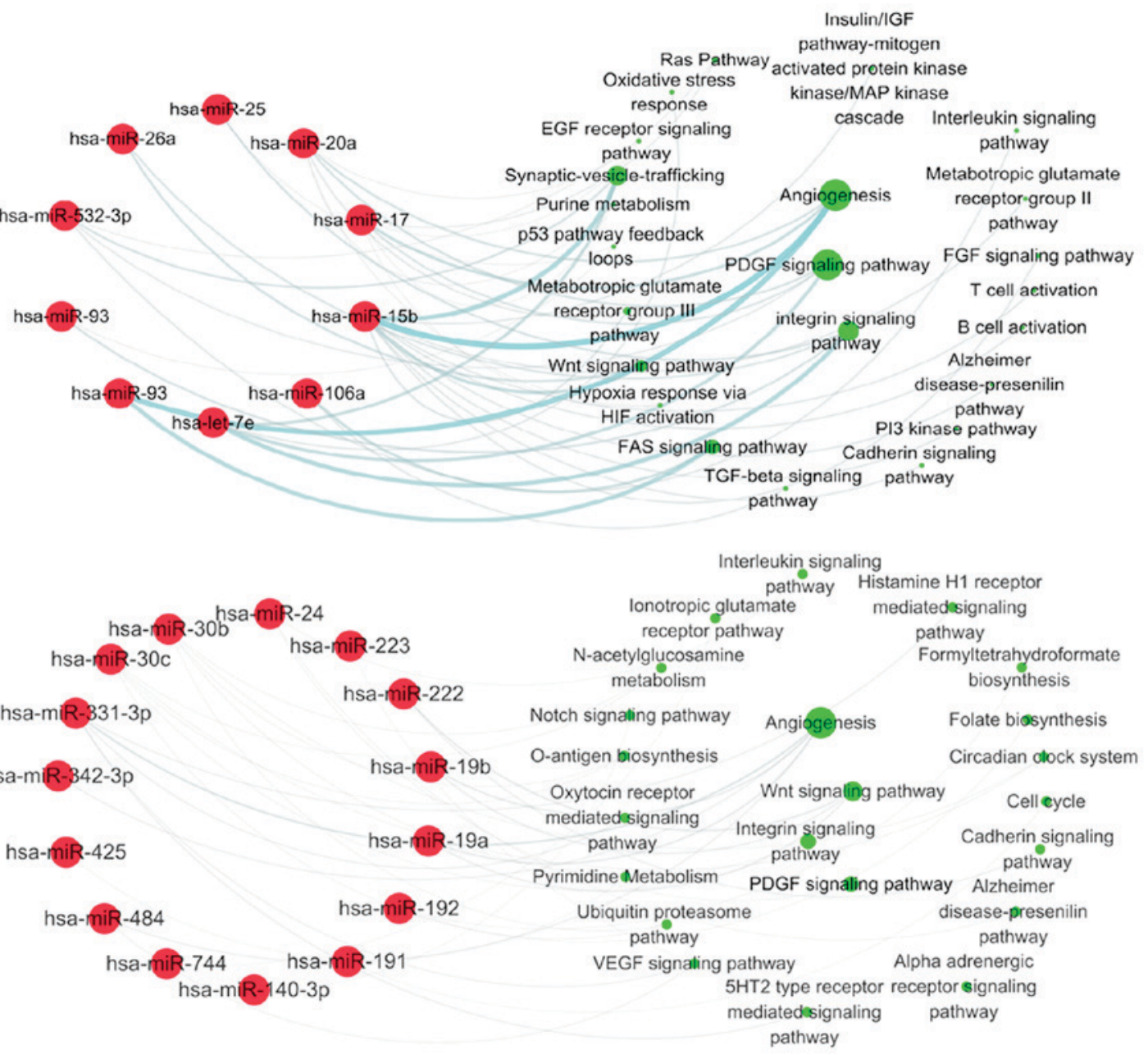

Figure 3. Potential signaling pathways targeted by upregulated miRNAs in the whole blood of statin-treated patients with UA compared with non-statin-treated UA patients. (A) Pathways associated with initially downregulated miRNAs in non-statin-treated patients, which were upregulated in statin-treated ones. (B) Pathways associated with nascent miRNAs in statin-treated patients. The pathways were clustered by DAVID based on the PANTHER pathways. The networks were generated by the Cytoscape tool. miRNA/miR, microRNA; UA, unstable angina; DAVID, Database for Annotation, Visualization and Integrated Discovery. 


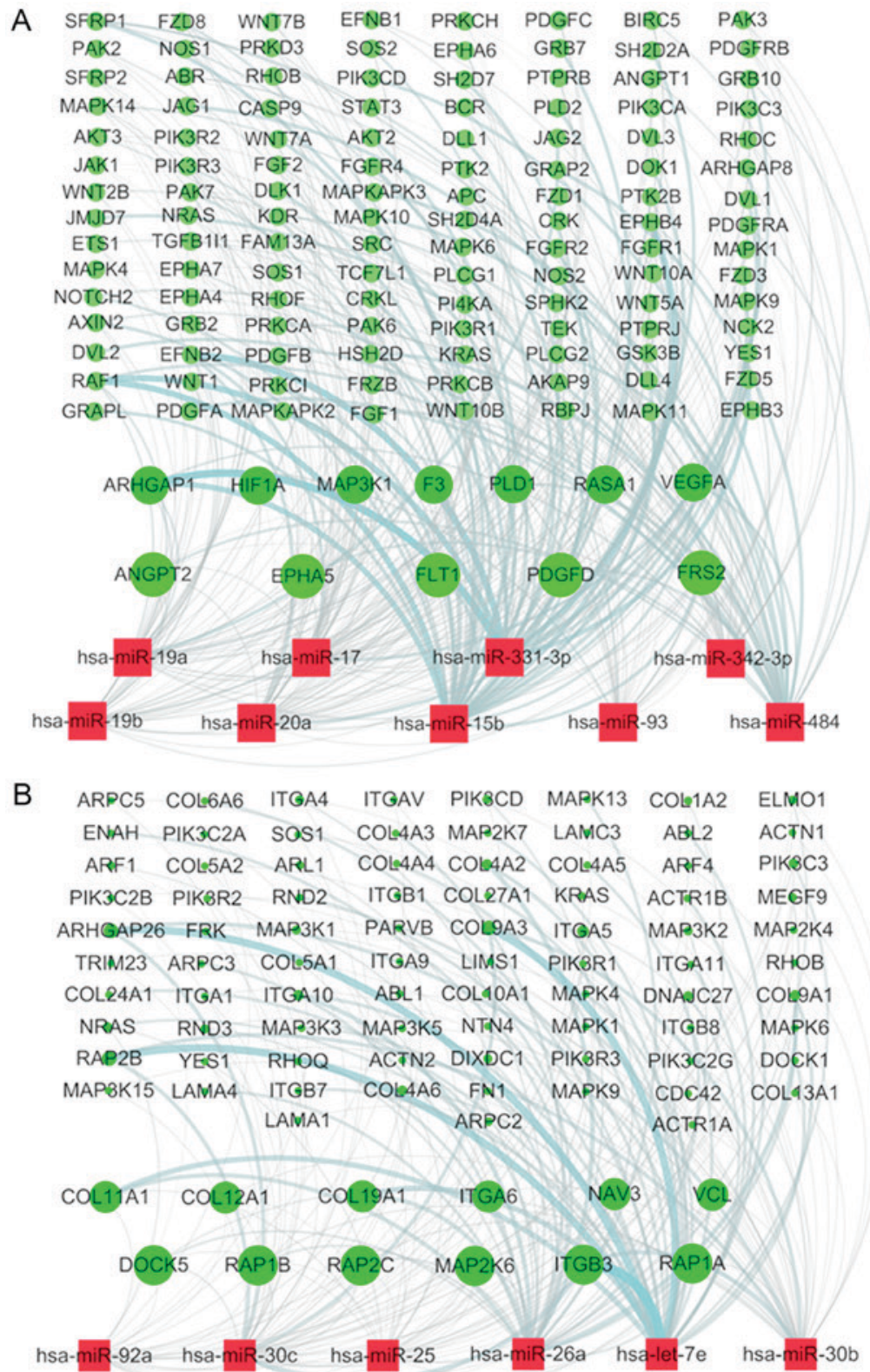

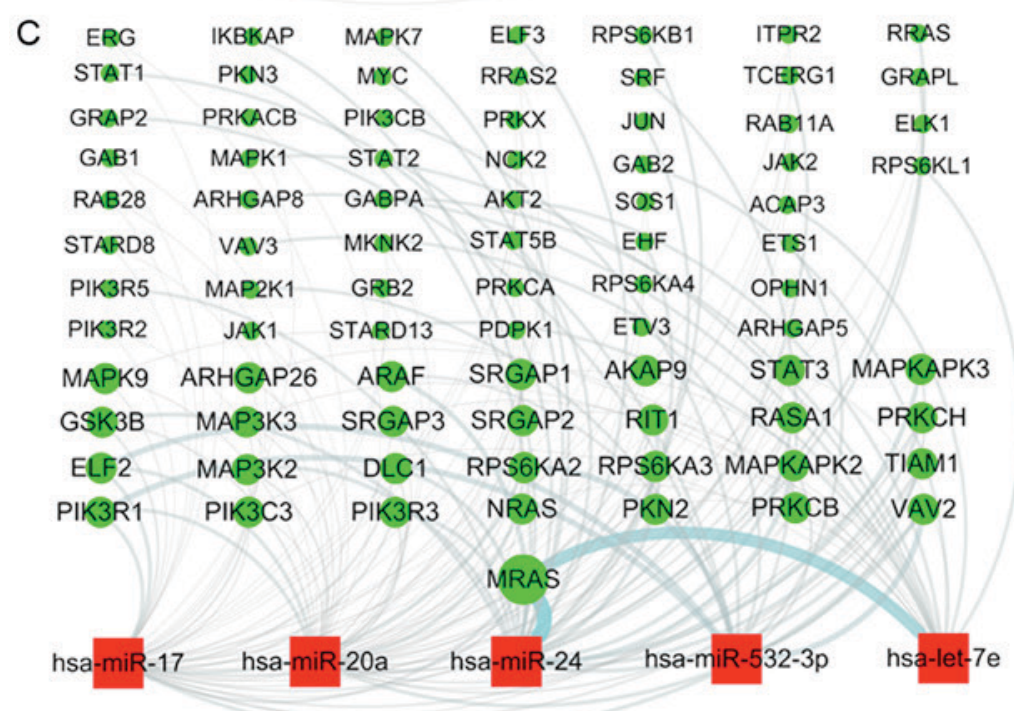

Figure 4. miRNA-genes network associated with the angiogenesis, integrin and PDGF signaling pathways. To obtain an overall view of the association between miRNAs and (A) angiogenesis, and the (B) integrin and (C) PDGF signaling pathways, the target genes of miRNAs involved in the three pathways were obtained and an miRNA-gene network was developed by the Cytoscape tool. miRNA/miR, microRNA; PDGF, platelet derived growth factor. 


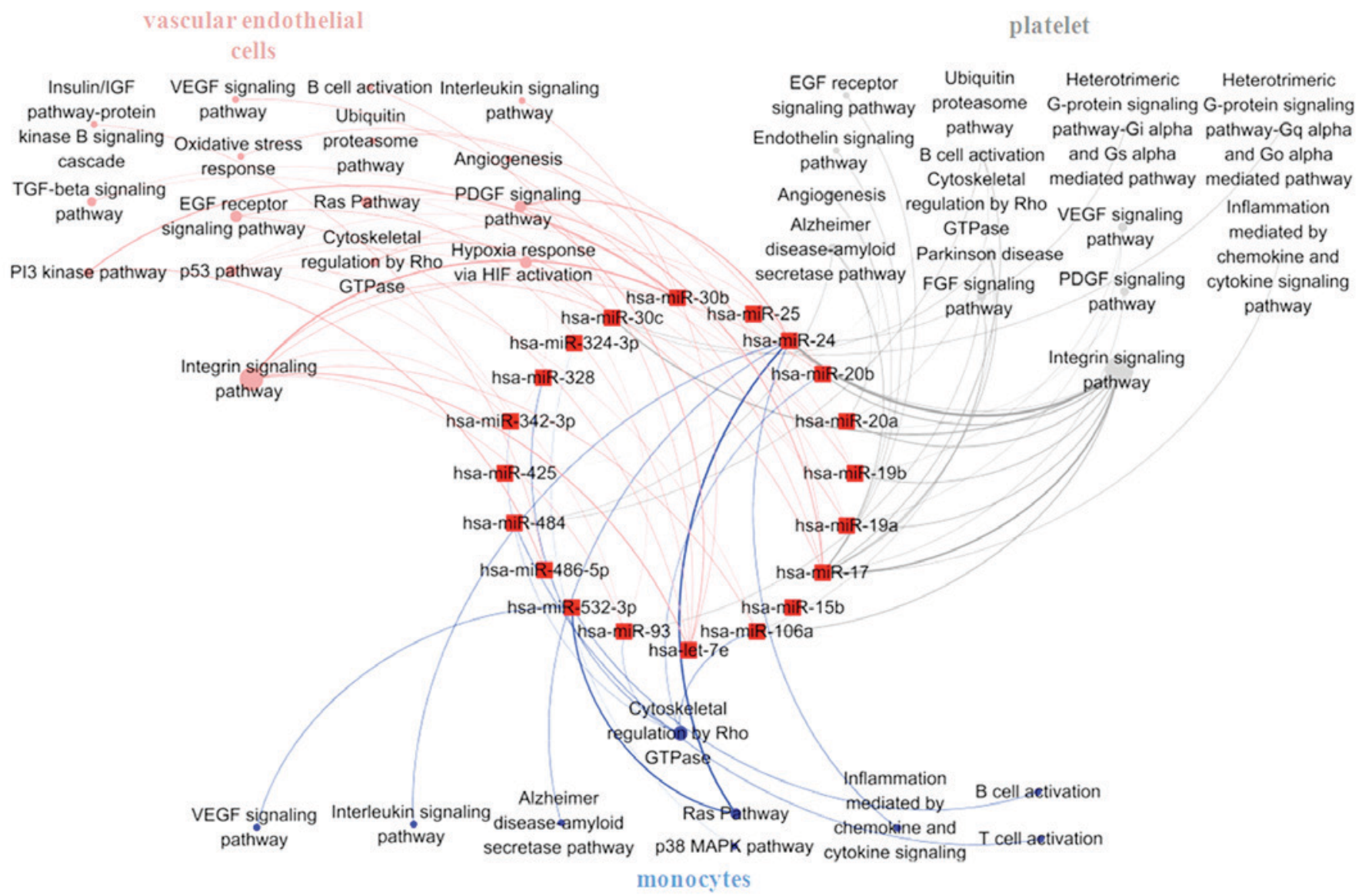

Figure 5. Target signaling pathways of upregulated miRNAs induced by statin in atherosclerosis-associated vascular endothelial cells, platelets and monocytes. Target genes of miRNAs were clustered based on CGAP SAGE (for monocytes and endothelial cells), or UP tissue-specific library in DAVID (for platelets), and categorized into three cell types. Specific genes in each cellular type were functionally clustered according to the PANTHER pathways. The two steps were performed in the DAVID platform and the network was generated by the Cytoscape tool. miRNA/miR, microRNA; DAVID, Database for Annotation, Visualization and Integrated Discovery.

observations, statins were demonstrated to inhibit inflammation/hypoxia-induced angiogenesis in endothelial cells or mice which may protect against plaque inflammatory angiogenesis and rupture (36-39), to reduce monocytes or hepatocellular carcinoma adhering to endothelium by interfering in the integrin signaling pathway $(40,41)$ and to suppress PDGF-mediated vascular smooth muscle proliferation and migration (42-44). A further target signaling pathways analysis of 33 upregulated miRNAs in atherosclerosis-associated vascular endothelial cells, platelets and monocytes demonstrated that statins primarily regulate the integrin signaling pathway in vascular endothelial cells and platelets, and mediate cytoskeletal regulation by the Rho GTPase pathway in monocytes, which was also confirmed to be associated with statins (45). The above results suggested that statins may facilitate atherosclerotic plaque stability through inhibiting angiogenesis, atherosclerosis-associated cell proliferation, monocyte migration, platelet adhesion and the coagulation cascade, mediated by circulating miRNAs.

The target genes of 33 upregulated miRNAs involved in the angiogenesis, integrin and PDGF signaling pathways were extracted. Bioinformatic analysis demonstrated that the targets in angiogenesis mainly included PDGF D, fms related tyrosine kinase 1, vascular endothelial growth factor receptor 1, fibroblast growth factor receptor substrate 2, ephrin type-a receptor
5 and angiopoietin-2. In the integrin signaling pathway they mainly included dedicator of cytokinesis protein 5 , dual specificity mitogen-activated protein kinase kinase 6 , integrin $\beta-3$, ras-related protein Rap-1A and ras-related protein Rap-2C. The PDGF signaling pathway included ras-related protein M-Ras, mitogen-activated protein kinase kinase kinase 2/3, protein kinase $\mathrm{C} \eta$ type, mitogen-activated protein kinase 9, rho GTPase-activating protein 26 , rho GTPase-activating protein $1 / 2 / 3$, rho GTPase-activating protein 7 , ras GTPaseactivating protein 1 , GTP-binding protein Rit 1 , ribosomal protein S6 kinase $\alpha-2 / 3$ and signal transducer and activator of transcription 3. These targets are all essential genes in the pathological process of plaque progression. Statins may reduce the expression of these genes by directly affecting miRNA levels in blood vessel cells and blood cells. Statins may also affect the release of miRNAs from the above cells, and subsequently enter into recipient cells and regulate their bioactivities by acting on their target genes via cell-cell communication (19).

In conclusion, the findings of the present study suggested that statins may exert protective effects on plaque stability by regulating the blood-borne miRNA network in UA patients. The definite role of statins in miRNA regulatory networks requires further validation through further biological experiments. 


\section{Acknowledgements}

The present study was supported by National Natural Science Foundation of China (grant nos. 81600340, 81270274,81470473, 81400265 and 81400264) and Beijing Municipal Science and Technology Project (grant no. D141100000114002).

\section{References}

1. Heidenreich PA, Trogdon JG, Khavjou OA, Butler J, Dracup K, Ezekowitz MD, Finkelstein EA, Hong Y, Johnston SC, Khera A, et al: Forecasting the future of cardiovascular disease in the United States: A policy statement from the American Heart Association. Circulation 123: 933-944, 2011.

2. LaRosa JC, Grundy SM, Waters DD, Shear C, Barter P, Fruchart JC, Gotto AM, Greten H, Kastelein JJ, Shepherd J, et al: Treating to New Targets (TNT) Investigators: Intensive lipid lowering with atorvastatin in patients with stable coronary disease. N Engl J Med 352: 1425-1435, 2005.

3. Cannon CP, Braunwald E, McCabe CH, Rader DJ, Rouleau JL, Belder R, Joyal SV, Hill KA, Pfeffer MA and Skene AM; Pravastatin or Atorvastatin Evaluation and Infection Therapy-Thrombolysis in Myocardial Infarction 22 Investigators: Intensive versus moderate lipid lowering with statins after acute coronary syndromes. N Engl J Med 350: 1495-1504, 2004.

4. Schwartz GG, Olsson AG, Ezekowitz MD, Ganz P, Oliver MF, Waters D, Zeiher A, Chaitman BR, Leslie S and Stern T; Myocardial Ischemia Reduction with Aggressive Cholesterol Lowering (MIRACL) Study Investigators: Effects of atorvastatin on early recurrent ischemic events in acute coronary syndromes: The MIRACL study: A randomized controlled trial. JAMA 285: 1711-1718, 2001.

5. Wright RS, Anderson JL, Adams CD, Bridges CR, Casey DE Jr, Ettinger SM, Fesmire FM, Ganiats TG, Jneid H, Lincoff AM, et al: 2011 ACCF/AHA focused update incorporated into the ACC/AHA 2007 Guidelines for the Management of Patients with Unstable Angina/Non-ST-Elevation Myocardial Infarction: A report of the American College of Cardiology Foundation/American Heart Association Task Force on Practice Guidelines developed in collaboration with the American Academy of Family Physicians, Society for Cardiovascular Angiography and Interventions, and the Society of Thoracic Surgeons. J Am Coll Cardiol 57: e215-e367, 2011.

6. Ray KK and Cannon CP: The potential relevance of the multiple lipid-independent (pleiotropic) effects of statins in the management of acute coronary syndromes. J Am Coll Cardiol 46: 1425-1433, 2005.

7. Angeli F, Reboldi G, Mazzotta G, Garofoli M, Cerasa MF and Verdecchia P: Statins in acute coronary syndrome: Very early initiation and benefits. Ther Adv Cardiovasc Dis 6: 163-174, 2012.

8. Chen H, Ren JY, Xing Y, Zhang WL, Liu X, Wu P, Wang RJ and Luo Y: Short-term withdrawal of simvastatin induces endothelial dysfunction in patients with coronary artery disease: A dose-response effect dependent on endothelial nitric oxide synthase. Int J Cardiol 131: 313-320, 2009.

9. Bartel DP: MicroRNAs: Genomics, biogenesis, mechanism, and function. Cell 116: 281-297, 2004.

10. Bartel DP: MicroRNAs: Target recognition and regulatory functions. Cell 136: 215-233, 2009.

11. Liu N and Olson EN: MicroRNA regulatory networks in cardiovascular development. Dev Cell 18: 510-525, 2010.

12. Ventura A and Jacks T: MicroRNAs and cancer: Short RNAs go a long way. Cell 136: 586-591, 2009.

13. Jacobsen A, Silber J, Harinath G, Huse JT, Schultz N and Sander C: Analysis of microRNA-target interactions across diverse cancer types. Nat Struct Mol Biol 20: 1325-1332, 2013.

14. Rottiers V and Näär AM: MicroRNAs in metabolism and metabolic disorders. Nat Rev Mol Cell Biol 13: 239-250, 2012.

15. Price NL, Ramírez CM and Fernández-Hernando C: Relevance of microRNA in metabolic diseases. Crit Rev Clin Lab Sci 51: 305-320, 2014

16. De Rosa S, Curcio A and Indolfi C: Emerging role of microRNAs in cardiovascular diseases. Circ J 78: 567-575, 2014.

17. Hagiwara S, Kantharidis P and Cooper ME: MicroRNA as biomarkers and regulator of cardiovascular development and disease. Curr Pharm Des 20: 2347-2370, 2014.
18. Creemers EE, Tijsen AJ and Pinto YM: Circulating microRNAs: Novel biomarkers and extracellular communicators in cardiovascular disease? Circ Res 110: 483-495, 2012.

19. Loyer X, Vion AC, Tedgui A and Boulanger CM: Microvesicles as cell-cell messengers in cardiovascular diseases. Circ Res 114: 345-353, 2014

20. Tabuchi T, Satoh M, Itoh T and Nakamura M: MicroRNA-34a regulates the longevity-associated protein SIRT1 in coronary artery disease: Effect of statins on SIRT1 and microRNA-34a expression. Clin Sci (Lond) 123: 161-171, 2012.

21. Sun HX, Zeng DY, Li RT, Pang RP, Yang H, Hu YL, Zhang Q, Jiang Y,Huang LY, Tang YB, et al: Essential role of microRNA-155 in regulating endothelium-dependent vasorelaxation by targeting endothelial nitric oxide synthase. Hypertension 60: 1407-1414, 2012.

22. Minami Y, Satoh M, Maesawa C, Takahashi Y, Tabuchi T, Itoh T and Nakamura M: Effect of atorvastatin on microRNA 221/222 expression in endothelial progenitor cells obtained from patients with coronary artery disease. Eur J Clin Invest 39: 359-367, 2009.

23. Zhang Q, Kandic I and Kutryk MJ: Dysregulation of angiogenesis-related microRNAs in endothelial progenitor cells from patients with coronary artery disease. Biochem Biophys Res Commun 405: 42-46, 2011.

24. Satoh M, Tabuchi T, Minami Y, Takahashi Y, Itoh T and Nakamura M: Expression of let-7i is associated with Toll-like receptor 4 signal in coronary artery disease: Effect of statins on let-7i and Toll-like receptor 4 signal. Immunobiology 217: 533-539, 2012.

25. Livak KJ and Schmittgen TD: Analysis of relative gene expression data using real-time quantitative PCR and the 2(-Delta Delta C(T)) Method. Methods 25: 402-408, 2001.

26. Leroyer AS, Isobe H, Lesèche G, Castier Y, Wassef M, Mallat Z, Binder BR, Tedgui A and Boulanger CM: Cellular origins and thrombogenic activity of microparticles isolated from human atherosclerotic plaques. J Am Coll Cardiol 49: 772-777, 2007.

27. Qiao Z, Ren J and Chen H: Simvastatin reduces expression and activity of lipoprotein-associated phospholipase $\mathrm{A}(2)$ in the aorta of hypercholesterolaemic atherosclerotic rabbits. J Int Med Res 37: 1029-1037, 2009.

28. Chen H, Ikeda U, Shimpo M, Ikeda M, Minota S and Shimada K: Fluvastatin upregulates inducible nitric oxide synthase expression in cytokine-stimulated rat vascular smooth muscle cells. Hypertension 36: 923-928, 2000.

29. Yahagi K, Kolodgie FD, Otsuka F, Finn AV, Davis HR, Joner M and Virmani R: Pathophysiology of native coronary, vein graft, and in-stent atherosclerosis. Nat Rev Cardiol 13: 79-98, 2016.

30. Wu G, Cai J, Han Y, Chen J, Huang ZP, Chen C, Cai Y, Huang H, Yang Y, Liu Y, et al: LincRNA-p21 regulates neointima formation, vascular smooth muscle cell proliferation, apoptosis, and atherosclerosis by enhancing p53 activity. Circulation 130: 1452-1465, 2014.

31. Sayin VI, Khan OM, Pehlivanoglu LE, Staffas A, Ibrahim MX, Asplund A, Agren P, Nilton A, Bergström G, Bergo MO, et al: Loss of one copy of $\mathrm{Zfp} 148$ reduces lesional macrophage proliferation and atherosclerosis in mice by activating p53. Circ Res 115: 781-789, 2014.

32. Huang CK, Pang H, Wang L, Niu Y, Luo J, Chang E, Sparks JD, Lee SO and Chang C: New therapy via targeting androgen receptor in monocytes/macrophages to battleatherosclerosis. Hypertension 63: 1345-1353, 2014.

33. Karshovska E, Zhao Z, Blanchet X, Schmitt MM, Bidzhekov K, Soehnlein O, von Hundelshausen P, Mattheij NJ, Cosemans JM, Megens RT, et al: Hyperreactivity of junctional adhesion molecule A-deficient platelets acceleratesatherosclerosis in hyperlipidemic mice. Circ Res 116: 587-599, 2015.

34. Ricci C and Ferri N: Naturally occurring PDGF receptor inhibitors with potential anti-atherosclerotic properties. Vascul Pharmacol 70: 1-7, 2015.

35. Heldin $\mathrm{CH}$ : Targeting the PDGF signaling pathway in the treatment of non-malignant diseases. J Neuroimmune Pharmacol 9: 69-79, 2014.

36. Li X, Liu X, Xu Y, He Y, Liu J and Xie M: Expression profile of apoptotic and proliferative proteins in hypoxic HUVEC treated with statins. Int J Oncol 46: 677-684, 2015.

37. Araújo FA, Rocha MA, Mendes JB, Andrade SP: Atorvastatin inhibits inflammatory angiogenesis in mice through down regulation of VEGF, TNF-alpha and TGF-beta1. Biomed Pharmacother 64: 29-34, 2010. 
38. Massaro M, Zampolli A, Scoditti E, Carluccio MA, Storelli C, Distante A and De Caterina R: Statins inhibit cyclooxygenase-2 and matrix metalloproteinase-9 in human endothelial cells: Anti-angiogenic actions possibly contributing to plaque stability. Cardiovasc Res 86: 311-320, 2010.

39. Weis M, Heeschen C, Glassford AJ and Cooke JP: Statins have biphasic effects on angiogenesis. Circulation 105: 739-745, 2002.

40. Weber C, Erl W, Weber KS and Weber PC: HMG-CoA reductase inhibitors decrease $\mathrm{CD} 11 \mathrm{~b}$ expression and CD11bdependent adhesion of monocytes to endothelium and reduce increased adhesiveness of monocytes isolated from patients with hypercholesterolemia. J Am Coll Cardiol 30: 1212-1217, 1997.

41. Relja B, Meder F, Wang M, Blaheta R, Henrich D, Marzi I and Lehnert M: Simvastatin modulates the adhesion and growth of hepatocellular carcinoma cells via decrease of integrin expression and ROCK. Int J Oncol 38: 879-885, 2011.
42. Chen S, Liu B, Kong D, Li S, Li C, Wang H and Sun Y: Atorvastatin calcium inhibits phenotypic modulation of PDGF-BB-induced VSMCs via down-regulation the Akt signaling pathway. PLoS One 10: e0122577, 2015.

43. Yoshikawa M, Nakamura K, Nagase S, Sakuragi S, Kusano KF, Matsubara $\mathrm{H}$ and Ohe T: Effects of combined treatment with angiotensin II type 1 receptor blocker and statin on stent restenosis. J Cardiovasc Pharmacol 53: 179-186, 2009.

44. Yang D, Yuan J, Liu G, Ling Z, Zeng H, Chen Y, Zhang Y, She Q and Zhou X: Angiotensin receptor blockers and statins could alleviate atrial fibrosis via regulating platelet-derived growth factor/Rac1/nuclear factor-kappa B Axis. Int J Med Sci 10: $812-824,2013$

45. Sawada N and Liao JK: Rho/Rho-associated coiled-coil forming kinase pathway as therapeutic targets for statins in atherosclerosis. Antioxid Redox Signal 20: 1251-1267, 2014. 